\title{
Phase II study on first-line treatment of NIVolumab in combination with folfoxiri/ bevacizumab in patients with Advanced COloRectal cancer RAS or BRAF mutated - NIVACOR trial (GOIRC-03-2018)
}

Angela Damato ${ }^{1,2^{*}}$ (D), Francesco lachetta ${ }^{1}$, Lorenzo Antonuzzo ${ }^{3}$, Guglielmo Nasti ${ }^{4}$, Francesca Bergamo ${ }^{5}$, Roberto Bordonaro ${ }^{6}$, Evaristo Maiello ${ }^{7}$, Alberto Zaniboni ${ }^{8}$, Giuseppe Tonini ${ }^{9}$, Alessandra Romagnani', Annalisa Berselli ${ }^{1}$, Nicola Normanno ${ }^{10}$ and Carmine Pinto ${ }^{1}$

\begin{abstract}
Background: FOLFOXIRI (fluorouracil, leucovorin, oxaliplatin, and irinotecan) plus bevacizumab has shown to be one of the therapeutic regimens in first line with the highest activity in patients (pts.) with metastatic colorectal cancer (mCRC) unselected for biomolecular alterations. Generally, tumors co-opt the programmed death-1/ligand 1 (PD-1/PDL1) signaling pathway as one key mechanism to evade immune surveillance. As today, anti-PD-1 monoclonal antibodies are FDA approved only for DNA mismatch repair deficient/microsatellite instability-high (MMRd/MSI-H), which represent only about 5\% among all mCRC. Nowadays, there are no data demonstrating anti PD-1 activity in proficient and stable disease (MMRp/MSS). A different target in MCRC is also the Vascular Endothelial Growth Factor A (VEGF-A), which acts on endothelial cells to stimulate angiogenesis. VEGF-A inhibition with bevacizumab has shown to increase the immune cell infiltration, providing a solid rationale for combining VEGF targeted agents with immune checkpoint inhibitors. Based on these evidences, we explore the combination of triplet chemotherapy (FOLFOXIRI) with bevacizumab and nivolumab in pts. with mCRC RAS/BRAF mutant regardless of microsatellite status.

(Continued on next page)
\end{abstract}

\footnotetext{
* Correspondence: angela.damato@ausl.re.it

${ }^{1}$ Medical Oncology Unit, Azienda Unità Sanitaria Locale - IRCCS di Reggio Emilia, Oncologia Medica, Dipartimento Oncologico e Tecnologie Avanzate, Viale Risorgimento 80, 42123 Reggio Emilia, Italy

${ }^{2}$ Department of Medical Biotechnologies, University of Siena, Strada delle Scotte 4, 53100 Siena, Italy

Full list of author information is available at the end of the article
}

(c) The Author(s). 2020 Open Access This article is licensed under a Creative Commons Attribution 4.0 International License, which permits use, sharing, adaptation, distribution and reproduction in any medium or format, as long as you give appropriate credit to the original author(s) and the source, provide a link to the Creative Commons licence, and indicate if changes were made. The images or other third party material in this article are included in the article's Creative Commons licence, unless indicated otherwise in a credit line to the material. If material is not included in the article's Creative Commons licence and your intended use is not permitted by statutory regulation or exceeds the permitted use, you will need to obtain permission directly from the copyright holder. To view a copy of this licence, visit http://creativecommons.org/licenses/by/4.0/ The Creative Commons Public Domain Dedication waiver (http://creativecommons.org/publicdomain/zero/1.0/) applies to the data made available in this article, unless otherwise stated in a credit line to the data. 
(Continued from previous page)

Methods/design: This is a prospective, open-label, multicentric phase II trial where pts. with mCRC RAS/BRAF mutated, in first line will receive nivolumab in combination with FOLFOXIRI/bevacizumab every 2 weeks for 8 cycles followed by maintenance with bevacizumab plus nivolumab every 2 weeks. Bevacizumab will be administered intravenously at dose of $5 \mathrm{mg} / \mathrm{kg}$ every 2 weeks and nivolumab intravenously as a flat dose of $240 \mathrm{mg}$ every 2 weeks. The primary endpoint is the overall response rate (ORR). This study hypothesis is that the treatment is able to improve the ORR from 66 to $80 \%$. Secondary endpoints include OS, safety, time to progression, duration of response. Collateral translational studies evaluate the i) tumor mutational burden, and ii) genetic alterations by circulating free DNA (cfDNA) obtained from plasma samples. The trial is open to enrollment, 9 of planned 70 pts. have been enrolled.

Trial registration: NIVACOR is registered at ClinicalTrials.gov: NCT04072198, August 28, 2019.

Keywords: Metastatic colorectal Cancer, First line therapy, Nivolumab, FOLFOXIRI Bevacizumab

\section{Background}

The colorectal cancer (CRC) is diagnosed at advanced stages in almost 50\%, and in this setting the 5-year survival rate is approximately of the $12 \%$ [1]. CRC is a heterogeneous tumor consisting of multiple genetic, genomic and epigenetic alterations, and this entails the stratification of the patients into different subgroups susceptible to different treatments. In 2015, a large-scale consortium reported four consensus molecular subtypes (CMS) of CRC described in MSI Immune (CMS1), Canonical (CMS2), Metabolic (CMS3), and Mesenchymal (CMS4) [2, 3], each with specific biomolecular and prognostic features.

In metastatic CRC (mCRC) the RAS gene (KRAS, $N R A S$ ) is mutated approximately in $50-55 \%$. Currently, detection of $R A S$ mutations is the only predictive marker of response to the anti-EGFR antibodies, cetuximab and panitumumab $[4,5]$.

The second potential biomarker in mCRC is $B R A F$, mutated in $5-11 \%$ of cases [6]. The BRAFV600E point mutation is the most common alteration and believed to be mutually exclusive with $K R A S$ exon 2 mutations [7]. Accordingly, several clinical trials have highlighted the negative prognostic role of the $B R A F$ mutation associated with high mortality [8].

In patients who harbor $R A S / B R A F$ mutant tumors, the addition of anti-vascular growth factor (VEGF) antibody to cytotoxic drugs based on fluorouracil/levofolinate/irinotecan or oxaliplatin, has become one of the standard treatments in first-line of $\mathrm{mCRC}$ [9].

Several randomized studies, have proved that the triplet of chemotherapy with fluorouracil/levofolinate/irinotecan/ oxaliplatin (FOLFOXIRI) combined to bevacizumab is more effective than doublet of chemotherapy plus bevacizumab, and this combination was well tolerated as firstline treatment in selected fit patients $[10,11]$. In the TRIBE study [9], a phase III study, in first-line setting the treatment with FOLFOXIRI plus bevacizumab improved the primary endpoint, progression-free survival (PFS), compared with FOLFIRI (fluorouracil, leucovorin, and irinotecan) plus bevacizumab (HR 0.75; 95\% CI 0.62-0.90; $p=0.003)$. A significant improvement and depth of tumor response associated with early tumor shrinkage assessed by Response Evaluation Criteria In Solid Tumors (RECI $\mathrm{ST}$ ) version 1.0, was also reported in experimental arm (FOLFOXIRI plus bevacizumab). Moreover, an advantage in terms of median overall survival (mOS) in FOLFOXIRI plus bevacizumab arm was revealed (29.8 months vs. 25.8 months; HR 0.80 , 95\% CI $0.65-0.98 ; p=0.03)$. The molecular sub-analysis of the TRIBE study showed a better $\mathrm{mOS}$ in $R A S$ and $B R A F$ wild-type subgroups compared to $R A S$ mutated and BRAF mutated subgroups (37.1 months vs. 25.6 months vs. 13.4 months), respectively [12].

In the VOLFI study [13], a phase II, patients affected by RAS wild type mCRC treated in first line with modifiedFOLFOXIRI (m-FOLFOXIRI) plus an anti-EGFR antibody, panitumumab, presented a significantly improved the ORR (87.3\%) compared to control arm (60.6\%) both investigator and centrally assessment (95\% CI, 1.61-12.38; $p=.004)$. No difference in PFS was found (9.7 months in both arms, HR 1.071; 95\%-CI 0.689-1.665, $p=0.76$ ), but a strong trend about enhanced mOS in the experimental arm has been reported (35.7 months vs. 29.8 months; HR: 0.67; 95\%-CI 0.41-1.11, $p=0.12$ ) [14].

Currently, a further tumor feature being studied and of the great interest is the description of immune landscape of the microenvironment in $\mathrm{mCRC}$, especially concern to microsatellite status. Most of tumors (8590\%) had a low-to-moderate mutation load and two main groups of CRCs were recognized: proficient in terms of mismatch repair mechanisms (MMRp) of DNA and microsatellite-stable (MSS). The minority are highly mutated with deficient mismatch-repair mechanisms (MMRd) relating to a microsatellite-instable phenotype (MSI, more accurately MSI-high [MSI-H]). This classification systems, MMRp/MSS vs. MMRd/MSI afford a way to stratify patients concerning to immunotherapy response $[15,16]$. Nowadays, the immunotherapy approach with anti-PD1/PD-L1 antibodies for $\mathrm{mCRC}$ has demonstrated efficacy only in MMRd/MSI tumor subgroups but no in MMRp/MSS tumors. Several phase I- 
II-III clinical trials have been conducted (Table 1) and others are still ongoing (Table 2) to establish the immunotherapeutic efficacy alone or in combination with other drugs, especially with chemotherapy.

Preclinical studies, have shown the close connection between tumor cells and the tumor microenvironment (TME) status, especially the surrounding milieu composed by the stroma, tumor-infiltrating lymphocytes, and lymphatic and vascular layers. In this context, endothelial cells play a key role in the extravasation of immune cells, influencing the arrangement of the tumor environment [38]. It is known that extremely inflamed tumors reflect poor tumor angiogenesis; however, highly vascularized tumors may conversely entail tumors with deprived immune infiltration. One of the biomolecules responsible for affecting the hematopoietic progenitor cell differentiation to dendritic cells (DCs) is the tumor-derived VEGF. DCs are the most efficient antigen presenting cells due to the peptide presentation of tumor antigens on the major histocompatibility complex (MHC) I and II molecules, eliciting Tcells by $\mathrm{B} 7$ molecule expression, against cancer antigens $[39,40]$. Active extravasation of leukocytes in the tumor stroma requires a series of events starting from the rolling, firm adhesion of leukocytes on endothelial cells, and leading to wandering into the interstitial areas. VEGF plays an essential role in this process as the blood vessels could present an obstacle to extravasation of immune cells in the interstitial space [41-43].
Additionally, VEGF inhibition by bevacizumab, involves a normalization of tumor vascularization rises the permeability to immune cell infiltration.

Given the strong preclinical rationale for combining VEGF inhibitors with immune checkpoint regulators, an increasing number of clinical trials are underway in several solid tumors including urothelial carcinoma [44, 45], metastatic renal cell carcinoma (mRCC) [46-48], and non-small cell lung cancer (NSCLC) [49-51], aiming to evaluate the anti-angiogenesis agents reinforce the benefit and durable responses afforded by anti- cytotoxic $\mathrm{T}$ lymphocyte associated protein-4 (CTLA4) and the PD-1/ PD-L1 agents.

It is essential to restore an immunological environment to sensitize mCRC to immune checkpoint inhibitors, to combine them with treatments that stimulate $\mathrm{T}$-cells as chemotherapy, although the molecular mechanisms of sensitization are still not clear. Preclinical models suggest that some chemotherapies can improve the immunotherapy efficacy $[52,53]$. The association of fluorouracil and oxaliplatin with immune checkpoint inhibitors in vivo could deplete Myeloid-derived Suppressor Cells (MDSCs) [54], and trigger an immunogenic arrangement of tumor cell death [55]. Dosset et al. [56], have investigated in two mouse models the use of FOLFOX in association with anti-PD-1 therapy. The combination induced a strong expression of PD- 1 on $\mathrm{CD}^{+}$TILs, and the IFN- $\gamma$ secreted by FOLFOX-induced $\mathrm{CD}^{+} \mathrm{T}$ cells leads PD-L1

Table 1 Clinical Trials in mCRC of immune-checkpoint inhibitors as single agents or in combination

\begin{tabular}{|c|c|c|c|c|c|c|c|}
\hline Drugs & Trial/Phase & Setting & Population & $\begin{array}{l}\text { ORR n/N } \\
(\%)\end{array}$ & $\begin{array}{l}\text { DCR n/N } \\
(\%)\end{array}$ & $\begin{array}{l}\text { PFS } \\
\text { (mo = months) }\end{array}$ & $\begin{array}{l}\text { OS } \\
\text { (mo = months })\end{array}$ \\
\hline Pembrolizumab [17] & $\begin{array}{l}\text { NCT01876511 } \\
\text { Phase II }\end{array}$ & $\geq 2 \mathrm{~L}$ & $\begin{array}{l}\text { MSI-H/ } \\
\text { dMMR }\end{array}$ & $\begin{array}{l}21 / 41 \\
(52)\end{array}$ & $\begin{array}{l}33 / 40 \\
(82)\end{array}$ & $\begin{array}{l}\text { 2-year }=59 \% \\
\text { mPFS NR }\end{array}$ & $\begin{array}{l}\text { 2-year }=85 \% \\
\text { mOS NR }\end{array}$ \\
\hline \multirow[t]{2}{*}{ Pembrolizumab $[18,19]$} & $\begin{array}{l}\text { KEYNOTE-164 } \\
\text { Phase II }\end{array}$ & $\geq 2 \mathrm{~L}$ & $\begin{array}{l}\text { MSI-H/ } \\
\text { dMMR }\end{array}$ & $\begin{array}{l}21 / 63 \\
(33)\end{array}$ & $\begin{array}{l}36 / 63 \\
(57)\end{array}$ & $\begin{array}{l}12-\mathrm{mo}=41 \% \\
\mathrm{mPFS} 4.1 \mathrm{mo}(2.1 \\
-\mathrm{NR})\end{array}$ & $\begin{array}{l}12-\mathrm{mo}=76 \% \\
\mathrm{mOS} \mathrm{NR}(19.2 \\
-\mathrm{NR})\end{array}$ \\
\hline & & $\geq 3 L$ & $\begin{array}{l}\text { MSI-H/ } \\
\text { dMMR }\end{array}$ & $\begin{array}{l}17 / 61 \\
(28)\end{array}$ & $\begin{array}{l}31 / 61 \\
(51)\end{array}$ & $\begin{array}{l}12-\mathrm{mo}=34 \% \\
\text { mPFS } 2.3 \mathrm{mo} \\
(2.1-8.1)\end{array}$ & $\begin{array}{l}12-\mathrm{mo}=72 \% \\
\mathrm{mOS} N R\end{array}$ \\
\hline Pembrolizumab + mFOLFOX6 [20] & $\begin{array}{l}\text { NCT02375672 } \\
\text { Phase II }\end{array}$ & $1 \mathrm{~L}$ & $\begin{array}{l}\text { MSI- } \\
\text { unselected }\end{array}$ & $\begin{array}{l}12 / 30 \\
(40)\end{array}$ & $\begin{array}{l}23 / 30 \\
(77)\end{array}$ & $\begin{array}{l}\text { PFS not reported } \\
\text { MPFS } 16.9 \text { mo } \\
(7.4,16.9)\end{array}$ & $\begin{array}{l}\text { OS not } \\
\text { reported } \\
\text { mOS } 8.8 \text { mo } \\
(18.3-\mathrm{NE})\end{array}$ \\
\hline Nivolumab [21] & $\begin{array}{l}\text { CheckMate } 142 \\
\text { Phase II }\end{array}$ & $\geq 2 L$ & $\begin{array}{l}\text { MSI-H/ } \\
\text { dMMR }\end{array}$ & $\begin{array}{l}23 / 74 \\
(31)\end{array}$ & $\begin{array}{l}51 / 74 \\
(69)\end{array}$ & $\begin{array}{l}12-\mathrm{mo}=50 \% \\
\mathrm{mPFS} 14.3 \mathrm{mo} \\
(4.3, \mathrm{NE})\end{array}$ & $\begin{array}{l}12-\mathrm{mo}=73 \% \\
\mathrm{mOS}, \mathrm{NR}(18.0 \\
\mathrm{NE})\end{array}$ \\
\hline Nivolumab + low dose Ipilimumab [22] & $\begin{array}{l}\text { ChackMate } 142 \\
\text { Phase II }\end{array}$ & $\geq 2 \mathrm{~L}$ & $\begin{array}{l}\text { MSI-H/ } \\
\text { dMMR }\end{array}$ & $\begin{array}{l}65 / 119 \\
(55)\end{array}$ & $\begin{array}{l}95 / 119 \\
(80)\end{array}$ & $\begin{array}{l}12-\mathrm{mo}=71 \% \\
\mathrm{mPFS} N R\end{array}$ & $\begin{array}{l}12-\mathrm{mo}=85 \% \\
\mathrm{mOS} N R\end{array}$ \\
\hline $\begin{array}{l}\text { Atezolizumab + bevacizumab and } \\
\text { fluoropyrimidine }[23]\end{array}$ & $\begin{array}{l}\text { NCT02291289 } \\
\text { Phase II }\end{array}$ & $\begin{array}{l}1 \mathrm{~L} \\
\text { (maintenance) }\end{array}$ & $\begin{array}{l}\text { MSI- } \\
\text { unselected }\end{array}$ & $\begin{array}{l}\text { Not } \\
\text { reported }\end{array}$ & $\begin{array}{l}\text { Not } \\
\text { reported }\end{array}$ & mPFS $7.2 \mathrm{mo}$ & mOS $22.1 \mathrm{mo}$ \\
\hline $\begin{array}{l}\text { Atezolizumab + FOLFOX + bevacizumab } \\
{[24]}\end{array}$ & $\begin{array}{l}\text { NCT01633970 } \\
\text { Phase lb }\end{array}$ & $\geq 2 \mathrm{~L}$ & $\begin{array}{l}\text { Oxaliplatin } \\
\text { naïve }\end{array}$ & $9 / 25(31)$ & $\begin{array}{l}\text { Not } \\
\text { reported }\end{array}$ & Not reported & Not reported \\
\hline Atezolizumab + bevacizumab [25] & $\begin{array}{l}\text { NCT01633970 } \\
\text { Phase I }\end{array}$ & $\geq 2 \mathrm{~L}$ & $\begin{array}{l}\text { MSI-H/ } \\
\text { dMMR }\end{array}$ & $4 / 10(40)$ & $9 / 10(90)$ & $\begin{array}{l}\text { MPFS NR }(1.5- \\
21.9)\end{array}$ & $\begin{array}{l}\operatorname{mOS} N R(2.6- \\
23.7)\end{array}$ \\
\hline
\end{tabular}

*ORR Overall response rate, PFS Progression free survival, OS Overall survival, NE Not estimable, NR Not reached, $m$ Median 
Table 2 Clinical Trials ongoing in mCRC of immune-checkpoint inhibitors as single agents or in combination with chemotherapy

\begin{tabular}{|c|c|c|c|c|}
\hline Drugs & Trial & Setting & $\begin{array}{l}\text { MSI/MSS status } \\
\text { population }\end{array}$ & Primary Endpoint \\
\hline Nivolumab + standard therapy vs standard therapy [26] & $\begin{array}{l}\text { CheckMate 9X8 } \\
\text { NCT03414983 } \\
\text { Phase I//II }\end{array}$ & $1 \mathrm{~L}$ & unselected & PFS \\
\hline $\begin{array}{l}\text { Nivolumab alone } \\
\text { Nivolumab in combination with other drugs [27] }\end{array}$ & $\begin{array}{l}\text { CheckMate } 142 \\
\text { NCT02060188 } \\
\text { Phase II }\end{array}$ & $1 \mathrm{~L}$ & MSI/MSS & $\begin{array}{l}\text { ORR by } \\
\text { investigators }\end{array}$ \\
\hline Nivolumab + Ipilimumab + Temozolomide [28] & $\begin{array}{l}\text { NCT03832621 } \\
\text { Phase II }\end{array}$ & $1 \mathrm{~L}$ & $\begin{array}{l}\text { MSS } \\
\text { MGMT silenced }\end{array}$ & 8-months PFS \\
\hline $\begin{array}{l}\text { Nivolumab } \\
\text { Nivolumab + Ipilimumab or standard therapy [29] }\end{array}$ & $\begin{array}{l}\text { NCT04008030 } \\
\text { Phase III }\end{array}$ & $1 \mathrm{~L}$ & MSI-H/MMRd & PFS \\
\hline Pembrolizumab vs standard therapy $[30]$ & $\begin{array}{l}\text { KEYNOTE-177 } \\
\text { NCT02563002 } \\
\text { Phase III }\end{array}$ & $1 \mathrm{~L}$ & MSI-H/MMRd & PFS, OS \\
\hline Pembrolizumab + pemetrexed and oxaliplatin [31] & $\begin{array}{l}\text { NCT03626992 } \\
\text { Phase Ib }\end{array}$ & $2 \mathrm{~L}+$ & MSS & ORR \\
\hline $\begin{array}{l}\text { Atezolizumab vs atezolizumab + FOLFOX/bevacizumab vs FOLFOX/ } \\
\text { bevacizumab [32] }\end{array}$ & $\begin{array}{l}\text { COMMIT GI004/S1610 } \\
\text { NCT02997228 } \\
\text { Phase III }\end{array}$ & $1 \mathrm{~L}$ & MMRd & PFS \\
\hline Avelumab [33] & $\begin{array}{l}\text { NCT03150706 } \\
\text { Phase II }\end{array}$ & $2 \mathrm{~L}+$ & $\begin{array}{l}\text { MSI-H/MMRd } \\
\text { POLE }\end{array}$ & ORR \\
\hline Avelumab vs standard chemotherapy $+/-$ targeted therapy $[34]$ & $\begin{array}{l}\text { NCT03186326 } \\
\text { Phase II }\end{array}$ & $2 \mathrm{~L}$ & MSI & $\begin{array}{l}\text { PFS by central } \\
\text { review }\end{array}$ \\
\hline Durvalumab [35] & $\begin{array}{l}\text { NCT02227667 } \\
\text { Phase II }\end{array}$ & $3 \mathrm{~L}+$ & MSI-I/MMRd & $\begin{array}{l}\text { best response } \\
\text { rate }\end{array}$ \\
\hline Durvalumab [36] & $\begin{array}{l}\text { NCT03435107 } \\
\text { Phase II }\end{array}$ & $2 \mathrm{~L}$ & $\begin{array}{l}\text { MSI/MMRd } \\
\text { POLE }\end{array}$ & ORR \\
\hline Durvalumab plus tremelimumab + FOLFOX [37] & $\begin{array}{l}\text { NCT03202758 } \\
\text { Phase } \mathrm{lb} / \mathrm{II}\end{array}$ & $1 \mathrm{~L}$ & unselected & safety, PFS \\
\hline
\end{tabular}

*MGMT, O6-methylguanine-DNA methyltransferase; POLE, DNA polymerase epsilon, catalytic subunit

expression on tumor cells and this mechanism is considered as an adaptive immune resistance system to FOLFOX. In neoadjuvant setting, mCRC patients treated with FOLFOX showed an increased $\mathrm{CD} 8^{+}$cell infiltrate and tumor PD-L1 expression. Another chemotherapeutic drug, trifluridine/tipiracil (FTD/TPI), an antimetabolite agent used to treat chemo-refractory $\mathrm{mCRC}$, induced immunogenic arrangement of tumor cell death in vitro in MSS CT26 mouse colon carcinoma cell line, as well as in various human MSS colorectal cancer cell lines [57]. In vivo, the combination of FTD/TPI with oxaliplatin was able to induce immunogenic arrangement of tumor cell death, but not the single agents. Furthermore, the combination abolished type- 2 tumor-associated macrophages (TAM2), resulting in higher cytotoxic $\mathrm{CD}^{+} \mathrm{T}$-cell infiltration and activation. This effect was associated with tumor cells PD-L1 expression and PD-1 induction in $\mathrm{CD} 8^{+} \mathrm{T}$ cells, resulting in T-cell exhaustion.

Based on these preclinical and clinical data, there is sufficient evidence to explore the combination of chemotherapy with immunotherapy and antiangiogenetic inhibitors in pts. with mCRC RAS/ $B R A F$ mutated.

\section{Methods}

Protocol overview/study treatment

This is a prospective, open-label, multicentric phase II trial in which pts. with $R A S$ or $B R A F$ mutated will receive nivolumab in combination with FOLFOXIRI/bevacizumab as first line treatment. Study screening will take place within 28 days prior to initiation of study treatment. At screening, every patient must have local RAS/ $B R A F$ known status. A centralized review of $R A S / B R A F$ status will be performed.

Eligible pts. will be enrolled and begin treatment with FOLFOXIRI/bevacizumab plus nivolumab every 2 weeks for 8 cycles followed by maintenance with bevacizumab plus nivolumab every 2 weeks until disease progression, unacceptable toxicity or patient/physician decision. Bevacizumab will be administered intravenously at dose of $5 \mathrm{mg} / \mathrm{kg}$ every 2 weeks. Nivolumab will be administered intravenously at flat dose of $240 \mathrm{mg}$ every 2 weeks. 
FOLFOXIRI will be administered as $165 \mathrm{mg} / \mathrm{m}^{2}$ intravenous infusion of irinotecan for $60 \mathrm{~min}$, followed by an $85 \mathrm{mg} / \mathrm{m}^{2}$ intravenous infusion of oxaliplatin given concurrently with leucovorin at a dose of $200 \mathrm{mg} / \mathrm{m}^{2}$ for $120 \mathrm{~min}$, followed by a $3200 \mathrm{mg} / \mathrm{m}^{2}$ continuous infusion of fluorouracil for $48 \mathrm{~h}$ (Fig. 1).

During the protocol's treatment, pts. will be followed for safety based on Adverse Event (AE) assessments including vital signs, physical findings and clinical laboratory test results.

In order to guarantee the safety of pts., the enrolment will be stopped when the 10th patient will start treatment. An Independent Monitoring Committee will evaluate the safety data of these pts. and will decide if the study should be completed, amended or closed.

The efficacy will be evaluated by the investigator according to RECIST 1.1 criteria every 8 weeks during treatment, and then every 3 months for 3 years.

During the study baseline tumor blocks will be centrally analyzed to determinate MSI/MSS and PD-L1 status, inflammatory infiltrate through evaluation of high peri- and/or intra-tumor lymphocyte infiltration (TIL) using $\mathrm{CD}^{+}$and $\mathrm{CD}^{+}$TILs, tumor-associated macrophages (TAMs), tumor-associated neutrophils (TANs), and regulatory $\mathrm{T}$ cells (Tregs) well as the expression of marker of autophagy.

Following discontinuation of the treatment, safety assessments will be conducted 30 days after the last drug administration or until initiation of other anti-cancer therapy. Thereafter, pts. will be followed for disease progression (unless this has already occurred), serious AEs, anticancer therapy and survival. Follow-up will continue for up to 3 years.

A blood sample will be collected at baseline, prior to cycle 5, at the end of chemotherapy and at disease progression. Quality of life will be assessed at baseline, every 4 weeks during treatment and study discontinuation visit.
A list of participating centers is provided in Table 3.

\section{Inclusion criteria}

For inclusion in the study, all of the following inclusion criteria must be fulfilled: (i) histopathological confirmed colon adenocarcinoma; (ii) initially unresectable metastatic colorectal cancer not previously treated with chemotherapy for metastatic disease; (iii) assessment of $R A S$ and $B R A F$ status of the primary and/or secondary colon cancer on biopsies (mutant); (iv) age $\geq 18$ years and $\leq 75$ years; (v) ECOG performance status $0-1$; (vi) if dihydropyridine dehydrogenase (DPD) status is known it must be wild type; (vii) laboratory data including: white blood cell count $\geq 3 \times 10^{9} / \mathrm{L}$ with neutrophils $\geq 1.5 \times 10^{9} /$ L, platelet count $\geq 100 \times 10^{9} / \mathrm{L}$, hemoglobin $\geq 9 \mathrm{~g} / \mathrm{dL}(5,6$ $\mathrm{mmol} / \mathrm{l}$ ), total bilirubin $\leq 1.5 \times$ ULN (upper limit of normal), ASAT and ALAT $\leq 2.5 \times$ ULN, alkaline phosphatase $\leq 1.5 \times$ ULN, serum creatinine $\leq 1.5 \times$ ULN; (viii) signed written informed consent obtained prior to any study specific screening procedures.

\section{Exclusion criteria}

Patients are not eligible for this study if any of the following exclusion criteria apply: (i) prior chemotherapy, excluded pts. treated in neo/adjuvant setting at least 12 months before diagnosis of metastatic disease; (ii) radiotherapy to any site within 4 weeks before the study; (iii) evidence of bleeding diathesis or coagulopathy; (iv) uncontrolled hypertension and prior history of hypertensive crisis or hypertensive encephalopathy (v) systemic corticosteroids within 2 weeks of the first dose of nivolumab; (vi) diagnosis of immunodeficiency or is receiving systemic steroid therapy within 14 days prior to the first dose of trial treatment; (vii) active and untreated brain (CNS) metastases and/or carcinomatous meningitis or subjects with previously treated brain metastases may participate provided they are not using steroids for at least 7

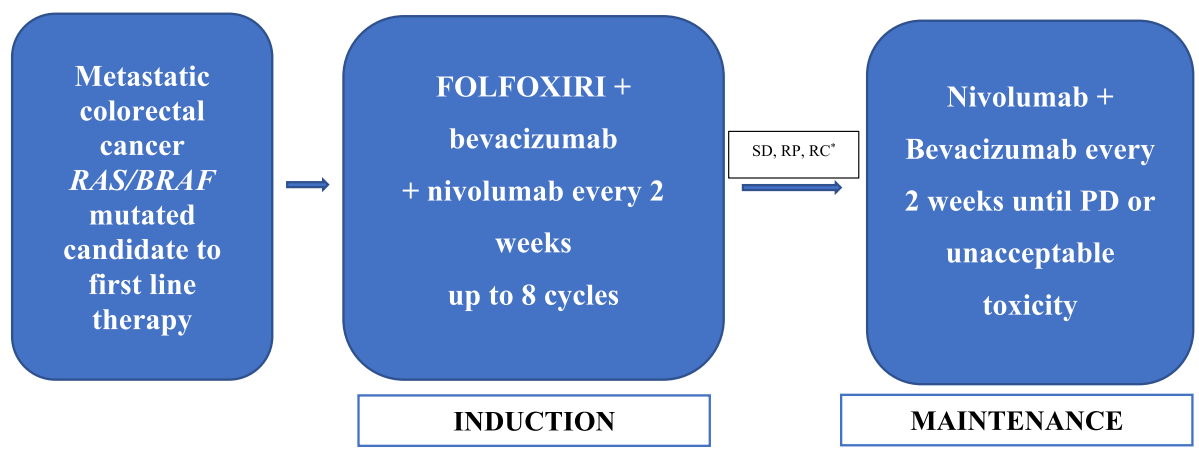

Primary Endpoint: Overall Response Rate (ORR) per investigator assessment (RECIST v1.1)

*SD: stable disease, RP: partial response, RC: complete response

Fig. 1 Study Design. Primary Endpoint: Overall Response Rate (ORR) per investigator assessment (RECIST v1.1). *SD: stable disease, RP: partial response, $\mathrm{RC}$ : complete response 
Table 3 Participating Centers

\begin{tabular}{lll}
\hline Principal Investigator & Site & City \\
\hline Carmine Pinto & Azienda USL - IRCCS Reggio Emilia & Reggio Emilia \\
Francesca Bergamo & Istituto Oncologico Veneto & Padova \\
Evaristo Maiello & Casa Sollievo della Sofferenza & San Giovanni Rotondo \\
Alberto Zaniboni & Fondazione Poliambulanza & Brescia \\
Lorenzo Antonuzzo & Azienda Ospedaliera Careggi & Firenze \\
Guglielmo Nasti & Istituto Nazionale Tumori di Napoli - IRCCS Pascale & Napoli \\
Giuseppe Tonini & Policlinico Universitario Campus Biomedico & Roma \\
Roberto Bordonaro & ARNAS Garibaldi - Azienda Ospedaliera di Rilievo Nazionale e di Alta Specializzazione Garibaldi Catania & C \\
\hline
\end{tabular}

days prior to trial treatment; (viii) evidence of interstitial lung disease, active non-infectious pneumonitis, or a history of grade 3 or greater pneumonitis; (ix) live vaccine within 30 days prior to the first dose of trial treatment; (x) significant vascular disease (e.g. aortic aneurysm requiring surgical repair or recent arterial thrombosis) within 6 months of study enrollment; (xi) history of abdominal fistula, gastrointestinal (GI) perforation, intra-abdominal abscess or active GI bleeding within 6 months prior to the first study treatment; (xii) pregnancy (absence to be confirmed by $ß$-hCG test) or breast-feeding period; (xiii) any significant disease which, in the investigator's opinion, would exclude the patient from the study.

\section{Study endpoints}

The present trial will determine if adding nivolumab to the first line therapy with FOLFOXIRI/bevacizumab is efficient in terms of response rate in mCRC RAS/BRAF mutated. To evaluate the Overall Response Rate (ORR), defined as complete response (CR), partial response (PR), and stable disease (SD), we will use RECIST version 1.1 criteria.

Secondary endpoints are the following: (i) safety assessment of the combination treatment with FOLFOXIRI/ bevacizumab plus nivolumab graded by National Cancer Institute (NCI) Common Terminology Criteria for Adverse Events (CTCAE) v. 4.03; (ii) OS defined as the time from beginning of the study-drug administration to the date of death from any cause; (iii) Time To Progression (TTP) defined as the time from beginning of the studydrug administration and the first date of documented progression, based on investigator assessment as per RECIST 1.1 criteria, or death due to any cause, whichever occurs first; (iv) the duration of response defined as the time between the first evidence of response (SD/PR/CR) and the date of documented progression or death due to any cause; (v) the quality of life of pts. determinate with the EORTC QLQ-C30 that consists of 30 questions that assess five aspects of patient functions (physical, emotional, role, cognitive, and social), three symptom scales (fatigue, nausea and vomiting, pain), global health and/or quality of life, and six single items (dyspnea, insomnia, appetite loss, constipation, diarrhea, financial difficulties) with a recall period of the previous week. Scale scores can be obtained for the multi-item scales.

The collateral study includes the TMB, MSI status and the role of genetic and molecular pattern analysis in relation to patient's outcome. Formalin-fixed and paraffinembedded (FFPE) tumor samples will be collected before starting fist-line therapy (at baseline), as primary and/or metastatic tumor tissue blocks or as 155 - $\mu \mathrm{m}$ unstained slides. The neoplastic cell content of each tumor sample will be assessed and in those cases with neoplastic cells $<50 \%$ a macro-dissection of the specimen will be performed, if possible. For all the pts. enrolled, venous blood will be obtained by standard phlebotomy technique from a peripheral access point or from a central line, by trained personnel. Blood samples will be collected at different points: at baseline, prior to 5 cycle, at the end of chemotherapy and at disease progression.

\section{Data collection and follow up}

Study drug administration occurs on Day 1 ( \pm 3 days) of each cycle. Each cycle is 14 days. Cycle 1 should occur within 3 days from registration of pts. All procedures during the study treatment must occur within 3 days prior to the administration, except for radiological assessment required for baseline within 28 days prior to initiation of the study treatment. The following assessments will be performed prior to each cycle every 2 weeks. All radiological assessments will be performed each 8 weeks ( \pm 1 week), regardless of the treatment cycle. CEA will be testing every 8 weeks with radiological assessments. The end of the study treatment visit should occur within 30 days after last dose of study treatment is administered. The post-treatment follow-up visits will occur every 3 months ( \pm 14 days) for 3 years (Table 4 ).

\section{Statistical analysis and sample size}

The primary objective of this study is to assess the ORR, defined as the best response recorded on the ITT population according to RECIST v1.1. In the TRIBE study, ORR 
Table 4 Study assessments

\begin{tabular}{|c|c|c|c|c|c|c|}
\hline Procedures & $\begin{array}{l}\text { Screening } \\
(-28 \text { days })\end{array}$ & $\begin{array}{l}\text { Cycle } 1,3,5,7^{\mathbf{a}} \\
\underline{\mathbf{+} 3 \text { days })}\end{array}$ & $\begin{array}{l}\text { Cycle } 2,4,6,8^{\mathbf{a}} \\
\underline{\mathbf{\pm} 3 \text { days })}\end{array}$ & $\begin{array}{l}\text { Maintenance }{ }^{\mathbf{a}} \\
( \pm 3 \text { days })\end{array}$ & End of treatment ${ }^{a}$ & $\begin{array}{l}\text { Post-treatment } \\
\text { Follow up }{ }^{\mathbf{a}} \\
\end{array}$ \\
\hline Signed informed consent & $x$ & & & & & \\
\hline Medical history and baseline conditions & $x$ & & & & & \\
\hline Physical examination & $x$ & $x$ & $x$ & $x$ & $x$ & \\
\hline Parameters ${ }^{\mathbf{b}}$ & $x$ & $x$ & $x$ & $x$ & $x$ & \\
\hline $\begin{array}{l}\text { Hematology and serum } \\
\text { chemistryc }^{c}\end{array}$ & $x$ & $x$ & $x$ & $x$ & $x$ & \\
\hline Protein dipstick & $x$ & $x$ & $x$ & $x$ & $x$ & \\
\hline Adverse Events & $x$ & $x$ & $x$ & $x$ & $x$ & \\
\hline Radiological assessment ${ }^{\mathrm{e}}, \mathrm{CEA}^{\mathrm{c}}$ & $x$ & $x$ & & $x$ & $x$ & \\
\hline QLQ-C30 questionnaire ${ }^{f}$ & $x$ & & $x$ & $x$ & $x$ & \\
\hline Blood sample ${ }^{g}$ & $x$ & & & & $x$ & \\
\hline FOLFOXIRI administration & & $x$ & $x$ & & & \\
\hline Nivolumab and Bevacizumab administration & & $x$ & $x$ & $x$ & & \\
\hline Survival follow-up & & & & & & $x$ \\
\hline
\end{tabular}

${ }^{a}$ Each cycle is 14 days. Study drugs administration occurs on day 1 (+/- 3 days) of each cycle. All clinical and laboratory assessments must occur within 3 days prior the administration. The end of treatment should occur within 30 days after last dose of study treatment. The post-treatment follow-up visit occur every 3 months (+/- 14 days) for 3 years

${ }^{b}$ Vital signs will include: weight, respiratory rate, pulse rate, temperature and systolic and diastolic blood pressure. At baseline height and BSA

'Hematology analysis (within 7 days before Cycle 1) consist of: hemoglobin, WBC and platelet count, BUN, creatinine, glucose, total bilirubin, sodium, potassium, calcium, AST, ALT, alkaline phosphatase, LDH, albumin. CEA will be tested every 8 weeks with radiological assessment. Amylase, lipase, TSH, FT3, FT4, will be done on cycle $2,4,6,8$

${ }^{d}$ If proteinuria is $2+$, should undergo a $24-\mathrm{h}$ urine collection and must demonstrate $1 \mathrm{~g}$ of protein $/ 24 \mathrm{~h}$

${ }^{e}$ Radiological assessment will be performed within 28 days prior to start of study treatment and every 8 weeks ( \pm 1 week), regardless cycle of treatment; in details, during chemotherapy phase prior to cycle 5 , at the end of chemotherapy (cycle 8)

${ }^{f} \mathrm{QLQ}-\mathrm{C} 30$ will be completed at baseline, at cycles 4 and 8 of chemotherapy phase, every 4 cycles thereafter and at end of treatment visit

${ }^{9}$ Blood sample will be collected at baseline, prior to cycle 5, at the end of chemotherapy and at time of progression

for pts. RAS/BRAF mutated treated in first line with FOLFOXIRI and bevacizumab regimen was $66 \%$ [11]. Our hypothesis is that FOLFOXIRI and bevacizumab regimen plus nivolumab is able to improve the ORR from 66 to $80 \%$. An ORR of $80 \%$ is considered enough valuable to pursue this combination in a phase III trial.

The sample size was calculated using the A'Hern [19] modification of the original Fleming [20] one-stage design. Calculations were performed by the use of PASS Professional v.11.0.10 software [21].

The study requires 64 subjects to decide whether the proportion responding, $\mathrm{P}$, is less than or equal to 0,66 or greater than or equal to 0,80 . If the number of responses is 49 or more, the Hypothesis that $P<0,66$ is rejected with a target error rate of 0,05 and an actual error rate of 0,046. If the number of responses is 48 or less, the hypothesis that $P>0,800$ is rejected with a target error rate of 0,200 and an actual error rate of 0,197. A total 70 pts. will to be enrolled assuming $10 \%$ pts. discontinuation rate due to non-compliance or toxicity.

\section{Preliminary safety evaluation}

An Independent Monitoring Committee (IDMC) will review safety data 28 days after the inclusion of the 10th patient. Safety data, including demographics, adverse events, serious adverse events, and relevant laboratory data, will be reviewed.

The IDMC will provide a recommendation as to whether the study may continue, whether amendment(s) to the protocol should be implemented, or whether the study should be stopped. The final decision will rest with the Sponsor.

\section{Coordination}

Azienda Unità Sanitaria Locale di Reggio Emilia - IRCCS is responsible for the coordination and management of the study on behalf of Gruppo Oncologico Italiano Ricerca Clinica (G.O.I.R.C.) Cooperative Group.

\section{Discussion}

The binding of PD-L1 to PD-1 plays a central role in Tcell tolerance by hindering naive and effector T-cell responses. Clinical experience with checkpoint inhibitors has shown that tumors co-opt the PD-L1/PD-1 signaling pathway as one key mechanism to escape immune damage. Nivolumab, an anti-PD-1 monoclonal antibody may block tumor growth in different ways by targeting certain cells.

It's well known that chemotherapy makes the cancer more immunogenic, and more suitable for immunotherapy. 
Moreover, angiogenetic inhibitors could promote enhanced tumor T-cell infiltration causing in a reprogramming of the tumor microenvironment from immune-suppressive to immune-permissive status. Novel anti-PD-L1 drugs reinforce the action of the antiangiogenetic drugs when administered in combination.

Encouraging early indicators of efficacy have been detected with combination strategies using immunecheckpoint inhibitors and biological targeted therapies, such as axitinib in combination with pembrolizumab [46], and nivolumab in combination with sunitinib or pazopanib [47] in mRCC. In another phase $1 \mathrm{~b}$ study in mRCC, investigating the combination of bevacizumab and an anti -PD-L1, atezolizumab, increased intratumoral $\mathrm{CD}^{+}{ }^{+} \mathrm{T}$-cells and macrophages compared to bevacizumab alone, leading to an increase of MHC I expression, as well as Th-1 and $\mathrm{T}$ effector gene signatures in post treatment biopsies assessment [48]. Atezolizumab plus bevacizumab were examined in phase I, II and III studies. The safety of this combination resulted acceptable and AEs leading to treatment interruption were very low. In a phase III study, $40 \%$ of pts. treated with atezolizumab plus bevacizumab and $54 \%$ of pts. with sunitinib had grade 3-4 AEs; 12 and $8 \%$ of allgrade AEs led to discontinuation of treatment, respectively $[48,58,59]$.

Recent findings for enhancement in PFS using bevacizumab and atezolizumab in combination with carboplatin/paclitaxel in front-line lung cancer is a promising strategy, indorsing clinically meaningful and durable benefit for patients [49-51].

In a clinical trial conducted in melanoma pts., has been explored the combination of bevacizumab with anti-CTLA-4 inhibitor, ipilimumab, revealed widespread morphological modifications in $\mathrm{CD} 1^{+}$endothelial cells and an extensive tumor penetration of immune cells post-treatment including $\mathrm{CD}^{+}$cells and $\mathrm{CD} 163^{+}$macrophages in comparison to ipilimumab treatment alone, thus demonstrating that the combination of anti-VEGF and anti-CTLA-4 inhibitors has the ability to promote immune cell access in the TME [60].

A recent phase III study revealed that in pts. with NSCLC, atezolizumab in addition to bevacizumab plus carboplatin and paclitaxel (ABCP) in 692 pts. with advanced non-squamous NSCLC improve OS (19.2 months vs. 14.7 months; HR 0.78 ; $95 \%$ CI, 0.64 to $0.96 ; p=0.02$ ) [51]. The safety profile of $\mathrm{ABCP}$ was consistent with safety profiles of each drugs and AEs occurred in $94.4 \%$ vs. $95.4 \%$ in $\mathrm{ABCP}$ and $\mathrm{BCP}$ control group, respectively. The most common grade 3 or 4 AEs were febrile neutropenia, and hypertension, and related serious AEs were noticed in 25.4 and $19.3 \%$ in the ABCP and BCP groups, respectively. The immune-related AEs (irAEs) grade 1 or 2 occurred in $77.4 \%$ of the $\mathrm{ABCP}$ group, and the treatment-related deaths occurred in $2.8 \%$ of the $\mathrm{ABCP}$ group [51].

In mCRC, a phase Ib study examined the safety and efficacy of atezolizumab plus bevacizumab (Arm A) with the dosage of atezolizumab $20 \mathrm{mg} / \mathrm{kg}$ q3w and bevacizumab $15 \mathrm{mg} / \mathrm{kg}$ q3w versus atezolizumab plus bevacizumab and mFOLFOX6 (Arm B) with atezolizumab $14 \mathrm{mg} / \mathrm{kg} \mathrm{q} 2 \mathrm{w}$, bevacizumab $10 \mathrm{mg} / \mathrm{kg} \mathrm{q} 2 \mathrm{w}$, and mFOLFOX6 at standard doses. The safety profile in Arm A showed a $64 \%$ of grade 3-4 AEs, while in Arm B, 73\% pts. had grade 3-4 AEs, especially hematological toxicity. The irAEs grade 3 and 4 were 7 and $20 \%$, respectively. The authors concluded that the addition of atezolizumab plus bevacizumab with or without FOLFOX was well tolerated without unexpected toxicities [61]. Efficacy data are not yet available.

In a phase II study, in $30 \mathrm{mCRC}$ pts., pembrolizumab combined with mFOLFOX6 in first line treatment showed an acceptable toxicity thought suggesting a trend towards an increase of neutropenia; in the initial cohort grade 3 and 4 neutropenia was described but after dose reduction of mFOLFOX6, rate of grade 3 and 4 toxicity was 36.7 and $13.2 \%$ with FOLFOX/pembrolizumab and pembrolizumab alone respectively. Best response was partial response in 15 pts. with $100 \%$ of disease control rate (DCR) at 8 weeks. After 2 months of therapy, one patient with MMRd had surgical resection accounting complete pathological response. Moreover, the mPFS has not been reached [62].

In conclusion, we assume that there are sufficient evidences to support the combination of treatments with triplet chemotherapy (FOLFOXIRI), antibody anti-VEGF (bevacizumab), and immunotherapy (nivolumab, anti PD-1 antibody) in pts. with mCRC RAS/BRAF mutated, regardless to MMR status.

\section{Abbreviations}

RAS: Rat sarcoma viral oncogene homolog; BRAF: V-Raf murine sarcoma viral oncogene homolog B1; FOLFOXIRI: 5-Fluorouracil, Oxaliplatin, Irinotecan; mCRC: Metastatic Colorectal Cancer; PD-1/PD-L1: Programmed death-1/ligand 1; MMRd/MSI-H: Mismatch repair deficient/microsatellite instability-high; MMRp/MSS: Mismatch repair proficient/microsatellite stable; VEGF: Vascular Endothelial Growth Factor; CMS: Consensus molecular subtypes; FOLFOX: 5Fluorouracil, Oxaliplatin; CEA: Carcinoembryonic antigen; pts.: Patients; ECOG PS: Eastern Cooperative Oncology Group - performance status; NCI CTCAE: National Cancer Institute Common Terminology Criteria for Adverse Events; ORR: Overall Response Rate; CR: Complete Response; PR: Partial Response; SD: Stable Disease; OS: Overall Survival; PFS: Progression Free Survival; TTP: Time to Progression; RECIST: Response Evaluation Criteria in Solid Tumors; AE: Adverse Event; Cl: Confidence Interval; HR: Hazard Ratio; FTD/TPI: Trifluridine/tipiracil; ICD: Immunogenic cell death; G.O.I.R.C.: Gruppo Oncologico Italiano Ricerca Clinica

\section{Acknowledgements}

Not applicable.

\section{Authors' contributions}

$A D, F I$ and $C P$ participated in the design of the study and wrote the original protocol for the study. $A D$, and CP drafted the manuscript. $L A, G N, F B, R B$, $E M, A Z, G T, A R, A B$ and NN directly provided their contribution, read and approved the final manuscript. 


\section{Funding}

The present study is founded by Bristol-Myers Squibb S.r.l. thought unrestricted grant for Contract Research Organization (CRO) services.

\section{Availability of data and materials Not applicable.}

\section{Ethics approval and consent to participate}

This study is conducted in agreement with either the Declaration of Helsinki or the laws and regulations of the country, whichever provides the greatest protection of the patient. The protocol has been written, and the study is conducted according to the ICH Harmonized Tripartite Guideline for Good Clinical Practice. The study (Protocol version 2.0, January 14th 2019) was approved for all participating centers by AIFA, the Italian health authority (Agenzia Italiana del Farmaco) on February 8th 2019 and registered on August 28th 2019 at Clinicaltrials.gov (NCT04072198). IEC(s)/IRB(s) approved the submitted documents for each center. Company QBE Insurance was appointed by Gruppo Oncologico Italiano Ricerca Clinica (G.O.I.R.C.) for an insurance policy to provide patients for reimbursement to any injury associated with the study. Changes to eligibility criteria, outcomes, analysis or other important protocol modifications will be notified to the IEC/IRB for approval and will be forwarded to the Sponsor. Informed consent to study procedures before enrollment in the study was signed by all candidates; moreover, those will be informed about the study purpose, the activities involved, the expected duration, the potential risks and benefits by the investigators (or legally authorized representative).

\section{Consent for publication}

Not applicable.

\section{Competing interests}

The authors declare no conflict of interest.

\section{Author details}

${ }^{1}$ Medical Oncology Unit, Azienda Unità Sanitaria Locale - IRCCS di Reggio Emilia, Oncologia Medica, Dipartimento Oncologico e Tecnologie Avanzate, Viale Risorgimento 80, 42123 Reggio Emilia, Italy. ${ }^{2}$ Department of Medical Biotechnologies, University of Siena, Strada delle Scotte 4, 53100 Siena, Italy. ${ }^{3}$ Azienda Ospedaliero - Universitaria Careggi, Dipartimento di Oncologia Medica, Largo G. Alessandro Brambilla 3, 50134 Firenze, Italy. ${ }^{4}$ Istituto Nazionale Tumori IRCCS Fondazione G. Pascale, Dipartimento di Oncologia Addominale, Via Mariano Semmola 53, Napoli, Italy. ${ }^{5}$ Istituto Oncologico Veneto I.R.C.C.S., S.C. Oncologia Medica 1, Dipartimento di Oncologia Clinica e Sperimentale, Via Gattamelata 64, 35128 Padova, Italy. ${ }^{6}$ ARNAS Garibaldi Azienda Ospedaliera di Rilievo Nazionale e di Alta Specializzazione Garibaldi, U.O.C. Oncologia Medica, Via Palermo 636, 95122 Catania, Italy. ${ }^{7}$ Casa Sollievo della Sofferenza, Oncologia Medica, Dipartimento Onco-Ematologico, Viale Cappuccini 1, 71013 San Giovanni Rotondo, Italy. ${ }^{8}$ Fondazione Poliambulanza Istituto Ospedaliero, U.O. Oncologia, Dipartimento Oncologico, Vial Leonida Bissolati 57, 25124 Brescia, Italy. ${ }^{9}$ Policlinico Universitario Campus Bio-Medico, Oncologia Medica, Via Alvaro del Portillo 200, 00128 Roma, Italy. ${ }^{10}$ Istituto Nazionale Tumori IRCCS Fondazione G. Pascale, Dipartimento della Ricerca, Via Mariano Semmola 53, Napoli, Italy.

Received: 8 April 2020 Accepted: 6 August 2020

Published online: 31 August 2020

\section{References}

1. National Cancer Institute Surveillance, Epidemiology, and end results program. SEER Stat Fact Sheets: Colon and Rectum Cancer. https://seer. cancer.gov/statfacts/html/colorect.html. Accessed 3 Mar 2016.

2. Guinney J, Dienstmann R, Wang $X$, et al. The consensus molecular subtypes of colorectal cancer. Nat Med. 2015;21(11):1350-6.

3. Dienstmann R, Vermeulen L, Guinney J, et al. Consensus molecular subtypes and the evolution of precision medicine in colorectal cancer. Nat Rev Cancer. 2017:17:79-92.

4. Fiala O, Buchler T, Mohelnikova-Duchonova B, et al. G12V and G12A KRAS mutations are associated with poor outcome in patients with metastatic colorectal cancer treated with bevacizumab. Tumour Biol. 2016;37:6823-30.
5. Sorich MJ, Wiese MD, Rowland A, et al. Extended RAS mutations and anti-EGFR monoclonal antibody survival benefit in metastatic colorectal cancer: a metaanalysis of randomized, controlled trials. Ann Oncol. 2015;26(1):13-21.

6. Davies H, Bignell GR, Cox C, et al. Mutations of the BRAF gene in human cancer. Nature. 2002;417:949-54.

7. Prahallad A, Sun C, Huang S, et al. Unresponsiveness of colon cancer to BRAF(V600E) inhibition through feedback activation of EGFR. Nature. 2012; 483(7387):100-3.

8. Bokemeyer $C$, Van Cutsem E, Rougier $P$, et al. Addition of cetuximab to chemotherapy as first-line treatment for KRAS wild-type metastatic colorectal cancer: pooled analysis of the CRYSTAL and OPUS randomised clinical trials. Eur J Cancer. 2012;48(10):1466-75.

9. Loupakis F, Cremolini C, Masi G, et al. Initial therapy with FOLFOXIRI and bevacizumab for metastatic colorectal Cancer. N Engl J Med. 2014;371:1609-18.

10. Hurwitz H, Tan BR, Reeves JA, et al. Updated efficacy, safety, and biomarker analyses of STEAM, a randomized, open-label, phase II trial of sequential (s) and concurrent (c) FOLFOXIRI-bevacizumab (BV) vs FOLFOX-BV for first-line (1L) treatment (tx) of patients with metastatic colorectal cancer (mCRC). J Clin Oncol. 2017:35:657.

11. Schmoll HJ, Meinert FM, Cygon F, et al. "CHARTA": FOLFOX/bevacizumab vs. FOLFOXIRI/ bevacizumab in advanced colorectal cancer-Final results, prognostic and potentially predictive factors from the randomized Phase II trial of the AIO. J Clin Oncol. 2017:35(15 suppl):3533.

12. Cremolini C, Loupakis F, Antoniotti C, et al. FOLFOXIRI plus bevacizumab versus FOLFIRI plus bevacizumab as first-line treatment of patients with metastatic colorectal cancer: updated overall survival and molecular subgroup analyses of the open-label, phase 3 TRIBE study. Lancet Oncol. 2015;16(13):1306-15.

13. Modest DP, Martens UM, Riera-Knorrenschild J, et al. FOLFOXIRI Plus Panitumumab As First-Line Treatment of RAS Wild-Type Metastatic Colorectal Cancer: The Randomized, Open-Label, Phase II VOLFI Study (AIO KRK0109). J Clin Oncol. 2019:37(35):3401-11. https://doi.org/10.1200/JCO.19. 01340 .

14. Geissler M, Tannapfel A, Reinacher-Schick A, et al. Final results of the randomized phase II VOLFI trial (AIO- KRKO109): mFOLFOXIRI 1 Panitumumab versus FOLFOXIRI as first- line treatment in patients with RAS wild-type metastatic colorectal cancer (mCRC). Abstract PD-030. Ann Oncol. 2019:iv119. https://doi.org/10.1093/annonc/mdz156.

15. Mlecnik B, Bindea GG, Angell HK, et al. Integrative analyses of colorectal cancer show immunoscore is a stronger predictor of patient survival than microsatellite instability. Immunity. 2016;44(3):698-711.

16. Kim JM, Chen DS. Immune escape to PD-L1/PD-1 blockade: seven steps to success (or failure). Ann Oncol. 2016;27:1492-504.

17. Le DT, Durham JN, Smith KN, et al. Mismatch repair deficiency predicts response of solid tumors to PD-1 blockade. Science. 2017;357:409-13.

18. Le DT, Kavan P, Kim TW et al. KEYNOTE-164: Pembrolizumab for patients with advanced microsatellite instability high (MSI-H) colorectal cancer. Presented at: American Society of Clinical Oncology; June 1-5, 2018; Chicago, IL. 2018; abstract 3514.

19. Diaz L, Marabelle A, Kim TW et al. Efficacy of pembrolizumab in phase 2 KEYNOTE-164 and KEYNOTE-158 studies of microsatellite instability high cancers. Presented at: European Society for Medical Oncology; September 8-12, 2017; Madrid, Spain. 2017; abstract 386P.

20. Shahda S, Noonan AM, Bekaii-Saab TS, et al. A phase II study of pembrolizumab in combination with mFOLFOX6 for patients with advanced colorectal. Presented at: American Society of Clinical Oncology; June 2-6, 2017; Chicago. IL 2017; abstract 3541.

21. Overman MJ, McDermott R, Leach $J$, et al. Nivolumab in patients with metastatic DNA mismatch repair-deficient or microsatellite instability high colorectal cancer (CheckMate 142): an open-label, multicentre, phase 2 study. Lancet Oncol. 2017;18:1182-91.

22. Overman MJ, Lonardi S, Wong KYM, et al. Durable clinical benefit with nivolumab plus ipilimumab in DNA mismatch repair-deficient/ microsatellite instability-high metastatic colorectal cancer. J Clin Oncol. 2018;36:773-9.

23. Grothey A, Tabernero J, Arnold D et al. Fluoropyrimidine (FP) and bevacizumab + atezolizumab as first-line treatment for BRAFwt metastatic colorectal cancer: Findings from the MODUL trial of biomarker-driven maintenance. Presented at: European Society for Medical Oncology; October 19-23, 2018; Munich, Germany. 2018; abstract LBA19.

24. Hochster HS, Bendell JC, Cleary JM et al. Efficacy and safety of atezolizumab (atezo) and bevacizumab (bev) in a phase lb study of microsatellite 
instability (MSI)-high metastatic colorectal cancer (mCRC). Presented at: American Society of Clinical Oncology Gastrointestinal Cancers Symposium; January 19-21, 2017; San Francisco, CA. 2017; abstract 673.

25. Bendell JC, Powderly JD, Lieu CH et al. Safety and efficacy of MPDL320A (anti-PDL1) in combination with bevacizumab (bev)and/or FOLFOX in patients (pts) with metastatic colorectal cancer (mCRC). Presented at: American Society of Clinical Oncology; May 29 June, 2015; Chicago, IL 2015; abstract 704.

26. An Investigational immunotherapy study of Nivolumab with standard of care therapy vs standard of care therapy for first line treatment of colorectal cancer that has spread (CheckMate 9X8). https://clinicaltrials.gov/ct2/show/ NCT03414983.

27. An Investigational Immuno-therapy Study of Nivolumab, and Nivolumab in Combination With Other Anti-cancer Drugs, in Colon Cancer That Has Come Back or Has Spread (CheckMate142). https://clinicaltrials.gov/ct2/ show/NCT02060188.

28. NIVOLUMAB Plus IPILIMUMAB and TEMOZOLOMIDE in microsatellite stable, MGMT Silenced Metastatic Colorectal Cancer (MAYA) https://clinicaltrials. gov/ct2/show/NCT03832621.

29. A Study of Nivolumab, Nivolumab Plus Ipilimumab, or Investigator's Choice Chemotherapy for the Treatment of Patients With Deficient Mismatch Repair (dMMR)/Microsatellite Instability High (MSI-H) Metastatic Colorectal Cancer (mCRC) (CheckMate 8HW). https://linicaltrials.gov/ct2/show/NCT04008030.

30. Study of pembrolizumab (MK-3475) vs standard therapy in partecipants with microsatellite instability high (MSI-H) or mismatch repair deficient (dMMR) stage IV colorectal carcinoma (MK-3475-177/KEY-NOTE-177). https:// clinicaltrials.gov/ct2/show/NCT02563002.

31. Study of Pembrolizumab With Pemetrexed and Oxaliplatin in ChemoRefractory Metastatic Colorectal Cancer Patients. NCT03626922. https:// clinicaltrials.gov/ct2/show/NCT03626922.

32. Combination Chemotherapy, Bevacizumab, and/or Atezolizumab in Treating Patients With Deficient DNA Mismatch Repair Metastatic Colorectal Cancer, the COMMIT Study. https://clinicaltrials.gov/ct2/show/NCT02997228.

33. Avelumab for MSI-H or POLE Mutated Metastatic Colorectal Cancer. https:// clinicaltrials.gov/ct2/show/NCT03150706.

34. Standard Chemotherapy vs Immunotherapie in 2nd Line Treatment of MSI Colorectal Mestastatic Cancer (SAMCO). https://clinicaltrials.gov/ct2/show/ NCT03186326.

35. Evaluate the Efficacy of MEDI4736 in Immunological Subsets of Advanced Colorectal Cancer. https://clinicaltrials.gov/ct2/show/NCT02227667.

36. Durvalumab for MSI-H or POLE Mutated Metastatic Colorectal Cancer. https://clinicaltrials.gov/ct2/show/NCT03435107.

37. Fumet JD, Isambert N, Hervieu A, et al. Phase Ib/I trial evaluating the safety, tolerability and immunological activity of durvalumab (MEDI4736) (anti-PDL1) plus tremelimumab (anti-CTLA-4) combined with FOLFOX in patients with metastatic colorectal cancer. ESMO Open. 2018;3:e000375. https://doi. org/10.1136/esmoopen-2018-000375.

38. Oyama T, Ran S, Ishida T, et al. Vascular endothelial growth factor affects dendritic cell maturation through the inhibition of nuclear factor-kappa B activation in hemopoietic progenitor cells. J Immunol. 1998;160:1224-32.

39. Gabrilovich D, Ishida T, Oyama T, Ran S, et al. Vascular endothelial growth factor inhibits the development of dendritic cells and dramatically affects the differentiation of multiple hematopoietic lineages in vivo. Blood. 1998; 92:4150-66.

40. Huang Y, Yuan J, Righi E, et al. Vascular normalizing doses of antiangiogenic treatment reprogram the immunosuppressive tumor microenvironment and enhance immunotherapy, Proc. Natl Acad Sci U S A. 2012;109:17561-6.

41. Becht E, de Reynies A, Giraldo NA, Pilati C, et al. Immune and stromal classification of colorectal cancer is associated with molecular subtypes and relevant for precision immunotherapy. Clin Cancer Res. 2016;22:4057-66.

42. Tian $L$, Goldstein A, Wang $H$, Ching Lo $H$, et al. Mutual regulation of tumor vessel normalization and immunostimulatory reprogramming. Nature. 2017; 544(7649):250-4. https://doi.org/10.1038/nature21724.

43. Borgstrom P, Hughes GK, Hansell P, et al. Leukocyte adhesion in angiogenic blood vessels role of E-selectin, P-selectin, and beta2 integrin in lymphotoxin-mediated leukocyte recruitment in tumor microvessels. J Clin Invest. 1997;99(9):2246-53. https://doi.org/10.1172/JCl119399.

44. Balar AV, Galsky MD, Rosenberg JE, Powles T, et al. Atezolizumab as first-line treatment in cisplatin-ineligible patients with locally advanced and metastatic urothelial carcinoma: a single-arm, multicentre, phase 2 trial. Lancet. 2017;389(10064):67-76.
45. Rosenberg JE, Hoffman-Censits J, Powles T, et al. Atezolizumab in patients with locally advanced and metastatic urothelial carcinoma who have progressed following treatment with platinum-based chemotherapy: a single-arm, multicentre, phase 2 trial. Lancet. 2016;387(10031):1909-20.

46. Atkins MB, Plimack ER, Puzanov I, et al. Axitinib in combination with pembrolizumab in patients (pts) with advanced renal cell carcinoma (aRCC): preliminary safety and efficacy results. Annals of Oncology. 2016;27(6):26695. https://doi.org/10.1093/annonc/mdw373.

47. Amin AERP, R. Infante J, Ernstoff SM, et al. Nivolumab (anti-PD-1; BMS936558, ONO-4538) in combination with sunitinib or pazopanib in patients (pts) with metastatic renal cell carcinoma (mRCC). J Clin Oncol. 2014;32(15 suppl):5010. https://doi.org/10.1200/jco.2014.32.15_suppl.5010.

48. Wallin JC, Bendell R, Funke $\mathrm{M}$, et al. Atezolizumab in combination with bevacizumab enhances antigen-specific T-cell migration in metastatic renal cell carcinoma. Nat Commun. 2016;7:12624. https://doi.org/10.1038/ ncomms 12624 .

49. Fehrenbacher $L$, Spira A, Ballinger $M$, et al. Atezolizumab versus docetaxel for patients with previously treated non-small cell lung cancer (POPLAR): a multicentre, open-label, phase 2 randomised controlled trial. Lancet. 2016; 387(10030):1837-46. https://doi.org/10.1016/S0140-6736(16)00587-0.

50. Reck MS, Cappuzzo F, Orlandi F, et al. Primary PFS and safety analyses of a randomized Phase III study of carboplatin+paclitaxel + /- bevacizumab, with or without Atezolizumab in $1 \mathrm{~L}$ non squamous metastatic NSCLC (IMpower150). ESMO Immuno Oncology Congress. 2017; Abstract 204. https:/oncologypro.esmo.org/meeting-resources/esmo-immuno-oncologycongress-2017/Primary-PFS-and-safety-analyses-of-a-randomized-Phase-IIIstudy-of-carboplatin-paclitaxel-bevacizumab-with-or-without-atezolizumabin-1L-non-squamous-metastatic-NSCLC-IMpower150.

51. Socinski MA, Jotte RM, Cappuzzo F, et al. Atezolizumab for first-line treatment of metastatic nonsquamous NSCLC. N Engl J Med. 2018;378:2288301. https://doi.org/10.1056/NEJMoa1716948.

52. Galluzzi L, Buque A, Kepp O, et al. Immunological effects of conventional chemotherapy and targeted anticancer agents. Cancer Cell. 2015;28(6):690714. https://doi.org/10.1016/j.ccell.2015.10.012.

53. Pfirschke C, Engblom C, Rickelt S, et al. Immunogenic chemotherapy sensitizes tumors to checkpoint blockade therapy. Immunity. 2016;44(2):34354. https://doi.org/10.1016/j.immuni.2015.11.024.

54. Vincent J, Mignot G, Chalmin F, et al. F. 5-fluorouracil selectively kills tumorassociated myeloid-derived suppressor cells resulting in enhanced T celldependent antitumor immunity. Cancer Res. 2010;70(8):3052-61. https://doi. org/10.1158/0008-5472.CAN-09-3690.

55. Tesniere A, Schlemmer F, Boige V, et al. Immunogenic death of colon cancer cells treated with oxaliplatin. Oncogene. 2010;29(4):482-91. https:// doi.org/10.1038/onc.2009.356.

56. Dosset M, Vargas TR, Lagrange A, et al. PD-1/PD-L1 pathway: an adaptive immune resistance mechanism to immunogenic chemotherapy in colorectal cancer. ONCOIMMUNOLOGY. 2018;7(6):e1433981. https://doi.org/ 10.1080/2162402X.2018.1433981

57. Limagne $\mathrm{E}$, Thibaudin $\mathrm{M}$, Nuttin $\mathrm{L}$, et al. Trifluridine/Tipiracil plus Oxaliplatin improves PD-1 blockade in colorectal Cancer by inducing immunogenic Cella death and depleting macrophages. Cancer Immunol Res. 2019;7:195869. https://doi.org/10.1158/2326-6066.CIR-19-0228.

58. McDermott DF, Huseni MA, Atkins MB, et al. Clinical activity and molecular correlates of response to atezolizumab alone or in combination with bevacizumab versus sunitinib in renal cell carcinoma. Nat Med. 2018;24(6):749-57.

59. Motzer RJ, et al. IMmotion151: a randomized phase III study of atezolizumab plus bevacizumab vs. sunitinib in untreated metastatic renal cell carcinoma (mRCC). J Clin Oncol. 2018;36(6):578.

60. Hodi FS, Lawrance D, Lezcano C, et al. Bevacizumab plus Ipilimumab in patients with metastatic melanoma. Cancer Immunol Res. 2014;2(7):632-42.

61. Bendell JC, Powderly JD, Lieu CH, et al. Safety and efficacy of MPDL3280A (anti-PDL1) in combination with bevacizumab (bev) and/or FOLFOX in patients (pts) with metastatic colorectal cancer (mCRC). J Clin Oncol. 2015; 33(Suppl. S3):704

62. Shahda S, Noonan AM, Bekaii-Saab TS, et al. A phase II study of pembrolizumab in combination with mFOLFOX6 for patients with advanced colorectal cancer. J Clin Oncol. 2017;35(15):3541.

\section{Publisher's Note}

Springer Nature remains neutral with regard to jurisdictional claims in published maps and institutional affiliations. 